\title{
El Control Organizativo en las Organizaciones No Gubernamentales: un Estudio de la Realidad Española
}

\author{
Cristina Amélia Pereira de Carvalho
}

\section{ResUMO}

Este artigo discute a questão do exercício do controle nas organizações e o caráter evolutivo do conceito ao longo da evolução da sociedade. No entanto considera que as organizações nãogovernamentais (ONGs), por suas características particulares, exigem uma abordagem analítica específica e, nesse sentido, propõe um modelo de análise capaz de perceber a especificidade do mundo das organizações não-lucrativas e de voluntariado. A verificação empírica ocorre em quatro ONGs espanholas envolvidas, nos últimos anos, por um ambiente sujeito a grandes mudanças em razão do intenso crescimento e importância sofridos pelo setor. Os dados foram coletados por meio de entrevistas semi-estruturadas com dirigentes das ONGs, e aplicação de questionários a uma amostra aleatória de voluntários estáveis de cada organização. Os resultados confirmam a proposição teórica, ainda que as interferências do ambiente assinalem diferenças já esperadas.

Palavras-chaves: controle organizacional; ambiente institucional; organizações nãogovernamentais.

\begin{abstract}
This article discusses the issue of the practice of control in organizations and the evolutive character of the concept throughout the society's evolution. However, it considers that the nongovernmental organizations (NGOs), for their particular characteristics, demand a specific analytical approach and, in this sense, it proposes a model of analysis capable of perceiving the specificity of the world of the nonprofit and the voluntary organizations. The empirical verification occurs in four Spanish NGOs involved, in the latest years, by an environment which is subject to great changes due to the boom of growth and importance in the field. Data were collected through semi-structured interviews to NGOs' directors and through application of questionnaires to a random sample of stable volunteers of each organization. The results confirm the theoretical proposition although the environment interferences indicate differences that were already expected.
\end{abstract}

Key words: organizational control; institutional environment; nongovernmental organizations. 


\section{INTRODUCCIÓN}

La literatura especializada ha demostrado que el control de las estrategias de acción, del proceso de trabajo y de los individuos en las organizaciones son cuestiones que están más allá de posibles modas. Estos temas han merecido siempre una atención especial por parte de los estudiosos de las organizaciones desde los tiempos de la gestión científica hasta las innovaciones organizativas y gerenciales de la actualidad.

El estudio del control organizativo presenta tradicionalmente dos planteamientos predominantes: por un lado, la perspectiva política que interpreta las organizaciones como instituciones formales donde el ejercicio del poder representa el núcleo duro y el objetivo es el dominio de unos miembros sobre otros; por otro, la perspectiva que destaca la eficiencia, donde el control se afianza como un instrumento a través del cual la organización puede lograr sus metas.

En la literatura organizacional este tema fue, siempre, preferentemente, discutido en el ámbito de las organizaciones públicas y de las empresas privadas. Las particulares características de las organizaciones no gubernamentales (ONGs), su separación equidistante del Estado y del mercado, su alejamiento de los paradigmas economicistas y organizacionales, señalan un tipo de organización que es, en realidad, micro cosmo representativo de la sociedad compleja en la que está enmarcada y que ya exige un acercamiento teórico y empírico que considere sus especificidades.

La expansión del fenómeno del asociacionismo y de los nuevos movimientos sociales relacionados con el pacifismo, la defensa de los derechos humanos, el ecologismo, el feminismo y la cooperación internacional para el desarrollo, en España, resultan claves en la defensa de los valores culturales contrahegemónicos y alternativos, contrapuestos al Estado, al mercado y al capital, al poder y a la acumulación. La multiplicación de las ONGs, estructuras cristalizadas de esos movimientos, además de despertar el interés del Estado que percibe el papel destacado de esas entidades en la ejecución de algunas políticas públicas (DiMaggio y Anheier, 1990), sensibiliza los académicos para la necesidad de dedicar esfuerzos a la creación de un cuerpo teórico capaz de interpretar y analizar las especificidades del sector. Este texto pretende contribuir para eso, al trazar un modelo de análisis del control organizacional que es ejercido en las ONGs españolas ${ }^{(1)}$. 


\section{La Racionalización de la Sociedad y la Evolución de las Estructuras de Control}

La atención prioritaria al control en el análisis organizacional evidencia una clara inspiración weberiana y se inscribe en la idea de que el ejercicio del poder está en el fundamento de las organizaciones. Sin embargo, las nociones de entorno y cultura que pusieron de manifiesto, por un lado, la influencia de las relaciones de la organización con su medio y, por otro, el peso de los valores de los individuos en su comportamiento en el interior de las organizaciones, posibilitaron nuevas formas de comprensión de los mecanismos actuales de control en las organizaciones.

Hasta la etapa pré-capitalista del desarrollo social, los modelos de producción eran poco rigurosos en términos del control del producto generado y del proceso empleado. La aceleración del desarrollo del proceso de producción se manifiesta en la emergencia de la nueva dinámica del sistema fabril caracterizada por la separación del trabajador de los medios de producción y la concentración de mano de obra en las fábricas urbanas. La aparición de la alienación por un lado y de la división del trabajo, por otro, son consecuencias visibles que revolucionan el proceso de trabajo.

De acuerdo con Burris (1989) el control simple fue la forma que permitió, en esa época, la acumulación y la apropiación capitalista. Este tipo de control fue eficaz y predominante en la sociedad, y lo sigue siendo actualmente en la pequeña empresa, mientras la producción capitalista era conducida por la pequeña escala.

Con la aparición de las grandes empresas y la nueva organización del trabajo, la forma de control simple, hasta entonces predominante en la producción, entra en crisis. Formas alternativas a la supervisión personalizada, característica del control simple, surgen como consecuencia de esos cambios (Burris, 1989). Mientras que el control técnico inspirado en el funcionamiento de las máquinas es característico del modo de trabajo fabril, el control burocrático legitimado por las normas y la jerarquía organizacional, caracteriza las actividades administrativas y más cualificadas. La burocracia se configura, de este modo, como el primer modelo de organización que utiliza mecanismos indirectos y menos visibles de control, al inscribir la reglamentación de las actividades, la especialización, la jerarquía y la división del trabajo en un conjunto de mecanismos estructurales objetivos e impersonales.

En un tercer nivel surge el control profesional que, establecido sobre códigos éticos, la educación formalizada y el estatus de los grupos profesionales se adecua a las actividades no rutinarias. Deducimos, por lo tanto que, a nuevas formas de organización del trabajo se asocian formas nuevas de control del proceso productivo. 
Herman (1982) reúne estas formas bajo el modo hegemónico de control del proceso de trabajo, que se caracteriza fundamentalmente por el consentimiento y la legitimación entre los niveles de ejecución y la dirección, desestimando la coerción. El autor señala en ese sentido que "la esencia del modo hegemónico es que [...] el compromiso y la participación sustituyen a la alienación y la dominación como modus vivendi del proceso de control del trabajo" (Herman, 1982, p. 14). En la nueva tendencia se persiguen la discreción y la legitimidad para un sistema de producción cuestionado por promover una intensa explotación de los trabajadores.

La eficiencia del modo hegemónico de control es, por lo tanto, señalada en su capacidad de aumentar la ocultación de la plusvalía. Se trata de la llamada eficiencia cualitativa que, a diferencia de la eficiencia cuantitativa que solamente busca producir mas output por unidad de input, se refiere a "las técnicas del proceso de trabajo que son usadas para asegurar la reproducción de las condiciones que permiten una continuada extracción de la plusvalía" (Herman, 1982, p. 11). En opinión de Edwards (1980) ésta es la gran ventaja del control burocrático sobre el taylorismo y el fordismo.

Las perspectivas liberales interpretan el auge de estos rasgos como indicativo del potencial humanizador y liberador de la sociedad pós-industrial. Edwards (1980), sin embargo, advierte que estos rasgos del control hegemónico no se pueden confundir con la autonomía en el trabajo, y son, tan solo, una manera de discreción puesto que el modo de control hegemónico tiene como principal propósito internalizar el control en los trabajadores, lo que significa hacerlo menos visible. La invisibilidad es la seña en alza.

Pero la evolución de las estructuras de control no se ha estancado y los tipos de control técnico, burocrático y profesional han evolucionado hacia un modo de control tecnocrático (Burris, 1989), más complejo y fuertemente legitimado. En este último se enfatiza la importancia, muy actual, del conocimiento, de la pericia y de la igualdad de oportunidades en el empleo, en un contexto de competencias por lograr un puesto de trabajo acorde con las aptitudes de cada uno.

Refuérzase la idea que las estructuras de control representan y acompañan la propia evolución de la sociedad capitalista y de los sistemas de producción, pero también que no son modelos puros ni exclusivos de una etapa histórica. En la mayoría de las organizaciones, según Burris (1989), coexisten dos o más formas de control dependiendo frecuentemente de su adecuación al tipo de trabajo que se realiza en cada una de las subunidades.

En una línea de argumentación semejante, Perrow (1990) sugiere que el proceso de industrialización de la sociedad moderna provocó el pasaje de los tipos de 
control directo para los controles burocráticos y posteriormente hacia el control de las premisas cognitivas. Para este autor el problema que ocasiona la necesidad de adaptación de los mecanismos de control a las transformaciones que ocurren en el seno de las organizaciones, son los límites de la racionalidad humana que hacen indispensable el control de las premisas de la toma de decisiones. Así, bajo esta orientación, la burocracia busca formas nuevas y más eficientes de dominación en "los controles más sutiles y no reactivos de los canales de comunicación, el vocabulario organizacional, etc" (Perrow, 1990, p. 156).

De ese modo, los controles directos o reactivos se refieren a las órdenes, la vigilancia directa, la regulación que se establece. Frecuentemente son formas de control de coste elevado que son válidas en situaciones de crisis y que no añaden experiencia o formación para el subordinado. Los controles burocráticos a su vez, más eficientes que los primeros, se componen de la especialización, la estandarización y la jerarquía. En ellos disminuye el flujo de información disponible lo que se traduce en que "el subordinado dispone de menos oportunidades para tomar decisiones que maximicen los intereses personales antes que los intereses de la organización" (Perrow, 1990, p. 156). Por último, el autor sugiere los controles completamente proactivos que se ejercen a través del dominio sobre las premisas cognitivas subyacentes a la acción. Constituyen una forma de control más difícil de ejercer, pero con la cual se obtiene más eficacia ya que son restringidas, voluntariamente, la gama de estímulos y alternativas disponibles para el individuo. Son, por lo tanto, premisas interiorizadas por los miembros de las organizaciones, que rigen sus conductas y sus intereses y que ganan espacio en el ámbito organizacional.

\section{El Contexto como Dimensión Cultural del Control Organizacional}

La atención al entorno cultural ha adquirido en los últimos tiempos un espacio importante en el análisis organizacional en general y en el análisis de las estructuras de control en particular.

Por medio del análisis cultural de las organizaciones se ha revelado el significado simbólico de una gran parte de las acciones cotidianas. La acción organizada se ve configurada por los valores y los esquemas de significado compartidos en el interior de los diferentes grupos. Los mitos, alimentados por la cultura organizacional, las creencias, los rituales y los héroes se perfilan como las representaciones simbólicas de esa cultura. Según Meyer y Rowan (1992), en organizaciones que, por sus características específicas, sean muy sensibles a sus entornos institucionales, como son los casos de las universidades y las 
organizaciones de voluntariado, las estructuras parecen reflejar en alto grado los mitos y las normas de sus entornos institucionales en vez de las demandas técnicas de sus actividades de trabajo.

En la evolución que han sufrido las organizaciones, hemos reflejado un caminar del mundo del trabajo desde las formas directas y coercitivas de control hacia modelos flexibles y difusos. En el siglo XIX las empresas industriales fueron incorporando, una tras otra, las formas de poder disciplinario y de control directo que se demostraban más eficaces en la coordinación de las tareas y la dominación del modo de trabajo. Hardy y Clegg (1996) argumentan que esa asunción de las nuevas técnicas no ocurrió en base a un plan predeterminado, sino a través de las presiones de las innovaciones institucionales de cada momento y de una respuesta isomórfica de las organizaciones. Sin apenas dominar el conocimiento de los nuevos sistemas de control, las organizaciones seguían las prácticas que se demostraban más eficaces.

En las últimas décadas, la legitimidad cuestionada de los controles burocráticos parece haber generado un proceso semejante en lo tocante a la difusión de las formas de control difusas, que tienen su locus en el dominio de las premisas culturales y en la dimensión simbólica de las organizaciones.

Este recorrido significó la revelación de los procesos latentes de control, invisibles y discretos, que demuestran una eficacia superior. La cultura organizativa, a través del individuo-miembro de la organización que tiene interiorizados los valores compartidos por el colectivo, define los objetivos y el modus operandi "sin apenas necesidad de recurrir a controles directos" (Pérez Vilariño y Schoenherr, 1987, p. 125). Además, por medio de los lazos valorativos creados entre los individuos en el interior de las organizaciones, se fortalecen los mecanismos de compromiso e identidad con la organización.

Tener compromisos con una organización representa la creación de fuertes lazos para los individuos. Según Ahrne (1990) la relación entre los individuos y la sociedad se establece en términos de formas de pertenencia organizacional según los diferentes sectores organizacionales.

Cada uno de los modelos de pertenencia - parentesco, afiliación, propiedad e ciudadanía - modela, de un modo particular, los lazos que unen a los individuos en los diferentes espacios organizacionales, favoreciendo la demarcación de la posición social de cada uno y los mecanismos de control social que predominan. Implica, igualmente, en nuestro entender, en formas específicas de coordinación de las actividades y de ejercicio de la autoridad sobre los miembros de la organización.

Si entendemos las organizaciones de voluntariado como organizaciones normativas en las que el comportamiento de sus miembros está fuertemente basado 
en el compromiso establecido y en las cuales, como subraya Ruiz Olabuenaga (1995, p. 137), "la obediencia [...] se basa fundamentalmente en la internalización de las directrices aceptadas como legítimas", podremos inferir que los tipos de control de carácter cultural serán, en un principio, predominantes y fundamentales en la conducción de la acción organizativa.

En el planteamiento presentado por Scott (1995), el ejercicio del control en las organizaciones puede situarse a lo largo de un continuum sobre el cual se hallan diferentes niveles de conformidad. Bajo el enfoque regulativo del control se encontraría una conformidad superficial, que correspondería a lo que hemos llamado los controles directos. En ellos se inscriben las leyes que regulan las actividades y las sanciones que punen el no seguimiento de esas leyes, la vigilancia y las estrictas normas técnicas para la producción. El enfoque normativo del control organizacional supone un control estructural basado en la certificación, el reconocimiento y la titulación, elementos propios de tipos más discretos de control que se apoyan en la regulación y sujeción de las premisas de la toma de decisión. En el otro extremo del continuum está el enfoque cognitivo del control que obtiene una conformidad más profunda a través del auto-control y del control entre pares y exige fuertes lazos de compromiso. Esta forma correspondería a los controles discretos y difusos que se fundamentan en el dominio de las premisas culturales.

En su momento, Weber (1993) sugirió que las estructuras regulativas, observadas desde una perspectiva histórica, tienden a moverse hacia una base normativa. Las características particulares de las organizaciones de voluntariado y su supuesta gran permeabilidad con el entorno, nos lleva a suponer que esta base normativa a su vez apunta hacia una naturaleza cognitiva. Sin embargo, la complejidad y el tamaño alcanzado por las organizaciones modernas sugiere la probable convivencia de las tres concepciones en una misma organización (Scott, 1995), en diferentes niveles y/o subunidades, creando un modelo de control institucional más completo.

Las organizaciones formales, en especial las organizaciones del sector institucional donde el control se ejerce en base a las normas y las exigencias del entorno, reflejan, de algún modo, según Scott (1987), la influencia del contexto en el que están implicadas, de igual forma que las subunidades de una organización son afectadas por las características y la acción de la organización en su totalidad.

En este sentido, diferentes organizaciones en contextos institucionales distintos podrán variar las proporciones en las que cada forma de control se hace presente en la conformación organizativa. El desarrollo de las formas de dominación simbólica transfiere la racionalidad de los fines de la acción organizacional al dominio de una racionalidad más difusa y aparentemente anárquica (Ruiz Olaebuenaga, 1995), siendo éste el proceso que parece ocurrir en las organizaciones no gubernamentales. 
De este modo, en esta investigación, aceptamos el supuesto de que las variaciones en el sistema de normas y creencias que conforman la dimensión simbólica de la sociedad y la red de relaciones que componen el contexto institucional, pueden influenciar e interferir en las estrategias de control de las organizaciones.

Así, por ejemplo, cambios en las exigencias de las administraciones públicas y en la reglamentación jurídica de un sector de actividad podrían tener efectos en las estructuras de control, conduciendo a la sofisticación de las formas de legalización/ formalización de las organizaciones (nos referimos por ejemplo a la cantidad de registros exigidos, la necesidad de presupuestos, el control contábil y las auditorias etc) e generar cambios en sus esquemas organizativos. En resumen, las organizaciones institucionalizadas tenderían a traducir progresivamente, para sus parámetros de gestión, las características predominantes del contexto en el que están insertas.

\section{Un Modelo de Análisis del Control en las ONGs}

La estrecha interrelación entre la sociedad en un sentido general y la organización, transforma esta en un forum social donde se reproduce el enfrentamiento de las estructuras económicas, sociales y políticas que componen el sistema social en un determinado momento. Clegg y Dunkerley (1991, p. 485) consideran inevitable interpretar las organizaciones como un "locus de dominación de una forma específica de vida", en el que se articulan los materiales que deben ser transformados: el capital y el trabajo creador de los individuos.

Desde esta perspectiva, la organización es considerada un elemento del contexto social y por lo tanto reproductor de las macro estructuras de poder y control en las que está inserta (Morgan, 1997). En este escenario se entabla la lucha entre individuos o grupos para hacerse con el poder y el control sobre las acciones organizativas, a través de las ideas (conocimiento y toma de decisión) y las técnicas (planificación y tecnología) que las generan.

La proposición de que el control organizativo representa un eslabón entre la acción social y el análisis estructural nos conduce a elegir, como criterio taxonómico, el influjo que los procesos sociales básicos y las principales formas de racionalidad ejercen en la configuración de las estructuras de control, destacando, en éstas, las formas menos perceptibles y difusas de control. 
En esta línea de razonamiento está la tipología sugerida por Pérez Vilariño y Schoenherr (1987, p. 125), donde "constituye un imperativo que los miembros de los niveles más bajos no pongan en cuestión el objetivo último de la organización". Para lograrlo, señalan que la forma de eliminar este riesgo "es controlar las bases en que se apoyan las decisiones de los miembros" (Pérez Vilariño y Schoenherr, 1987). De este modo coinciden con Perrow (1990) en la trascendencia del control de las premisas cognitivas en las organizaciones.

En un primer lugar está el control directo, una forma completamente abierta que se ejerce mediante "la imposición de órdenes y la vigilancia expresa". Esta forma de control es adoptada por ejemplo en las llamadas organizaciones totales, en las cuales se incluyen las prisiones y los hospitales psiquiátricos; pero también en las fábricas, en las orígenes de la revolución industrial y todavía predomina hoy día en muchas plantas industriales. Esta forma directa de control se centra en las normas técnicas para la producción y se evidencia en la subordinación real del trabajo. Por estos motivos, puede participar en la configuración de control de organizaciones poco formalizadas que estén requiriendo mayor dominio sobre el proceso de trabajo.

En un segundo nivel está el control estructural que se produce en "los niveles más formalizados de la organización" y que, para los autores, representa un tipo de control discreto (unobtrusive). Perrow (1990), que discute también la convicción de que las estructuras organizacionales sean en realidad mecanismos formalizados de control, manifiesta que el control estructural está previamente establecido en las premisas que sostienen la toma de decisión. Éstas suelen ser en concreto las reglas, los criterios de selección de personal, la complejidad, la formalización, la centralización, entre otros aspectos que componen en su conjunto el aparato formal de la organización.

Por último está el control completamente difuso, un tipo ya perfectamente discreto (fully unobtrusive control) que se realiza sobre los presupuestos culturales de la toma de decisión en lugar de materializarse sobre los aspectos más concretos de este proceso. "Es un tipo de control más difícil de conseguir, pero mucho más eficaz", afirman Pérez Vilariño y Schoenherr (1987, p. 126), puesto que se ejerce en los fundamentos cognitivos de la organización, en sus procesos culturales. Éstos incluyen los rituales que cumplen un "importante papel de cohesión en cuanto celebraciones compartidas de la pertenencia común", y el vocabulario propio que "expresa y consolida el sentido de exclusividad en los miembros". Este tercer tipo de control está marcado por el enfoque cultural.

De este modo refuérzase la idea de la naturaleza evolutiva del control organizacional puesto que, se verifica una evolución del tipo de control directo hacia el tipo de control difuso, que parece acompañar la evolución de las formas 
de organización social, desde las organizaciones tayloristas cerradas en si mismas, hasta las organizaciones institucionalizadas que revelan permeabilidad al entorno. El control de tipo fordista, asentado en el acuerdo establecido entre empresario y empleado mediante un contrato de trabajo que asegura la remuneración de este último, tiende a ser sustituido, según Courpasson (1997), por nuevas formas de dominación que privilegian el control de los comportamientos individuales, del compromiso y de la lealtad con la organización.

\section{Cuadro 1: Tipologia de Control}

\begin{tabular}{||c|l|l|l|l||}
\hline Control & \multicolumn{1}{|c|}{ Medios } & \multicolumn{1}{|c||}{ Donde se ejerce } & \multicolumn{1}{c||}{$\begin{array}{c}\text { Modelos de } \\
\text { análisis }\end{array}$} & \multicolumn{1}{c||}{$\begin{array}{c}\text { Tipo de } \\
\text { empresa }\end{array}$} \\
\hline Directo & $\begin{array}{l}\text { Imposición de } \\
\text { órdenes } \\
\text { Vigilancia } \\
\text { expresa }\end{array}$ & $\begin{array}{l}\text { Organizaciones } \\
\text { totales } \\
\text { (cárceles) }\end{array}$ & Taylorismo & $\begin{array}{l}\text { Pequeñas } \\
\text { empresas }\end{array}$ \\
\hline Estructural & $\begin{array}{l}\text { Normas } \\
\text { Reglamentos } \\
\text { Jerarquía } \\
\text { Criterios de } \\
\text { selección }\end{array}$ & $\begin{array}{l}\text { Grandes } \\
\text { organizaciones } \\
\text { burocráticas } \\
\text { (industrias, servicios) }\end{array}$ & $\begin{array}{l}\text { Tipo ideal de } \\
\text { burocracia }\end{array}$ & $\begin{array}{l}\text { Grandes } \\
\text { empresas } \\
\text { capitalistas }\end{array}$ \\
\hline Difuso & $\begin{array}{l}\text { Presupuestos } \\
\text { culturales }\end{array}$ & $\begin{array}{l}\text { Organizaciones } \\
\text { normativas } \\
\text { (universidades, ONG) }\end{array}$ & $\begin{array}{l}\text { Anarquías } \\
\text { organizadas }\end{array}$ & $\begin{array}{l}\text { Organizaciones } \\
\text { institucionali- } \\
\text { zadas }\end{array}$ \\
\hline \hline
\end{tabular}

Fuente: elaboración propia a partir de la tipología diseñada por Pérez Vilariño y Schoenherr (1987).

La importancia creciente, en la sociedad burocrática, de los tipos invisibles, difusos o intuitivos de control frente a las formas directas, simples y estructurales sugiere la noción de la limitación flexible en la cual el individuo tiene aparentemente la posibilidad de elegir la calidad de su participación. En realidad, sin embargo, en el gobierno actual de las organizaciones, cualificado como una dominación suave, las evaluaciones, los mecanismos de promoción, las opciones de los directivos que el subordinado presencia en su vida diaria "ejercen un efecto de limitación sin violencia explícita, que va a guiar su conducta en numerosas circunstancias" (Courpasson, 1997, p. 55).

Con base en las formulaciones de Pérez Vilariño y Schoenherr (1987) y consistente con los aportes de Burris (1989), Perrow (1990) y Scott (1995), podemos visualizar un modelo de análisis en él cual cada estrategia de control es formada por una combinación diferente de los tres tipos de control.

De ese modo, organizaciones de tipo taylorista estarían preferencialmente subordinadas al control directo en el cual se privilegian la claridad de órdenes, la rigidez de los horarios y la supervisión directa de las tareas entre otros factores. 
Figura 1: Estrategia de Control en Organizaciones Tayloristas

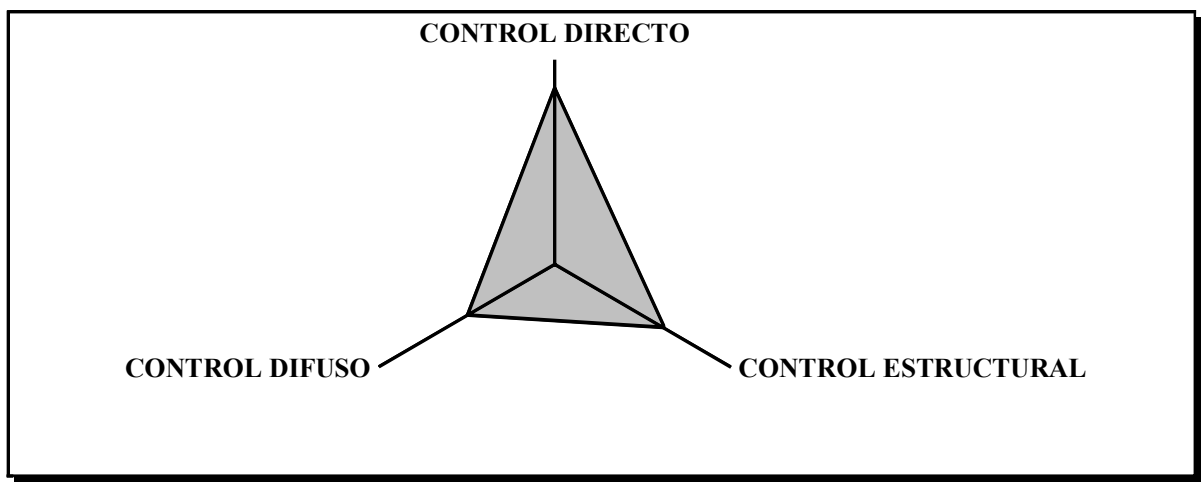

Fuente: elaboración propia.

A su vez, las organizaciones burocráticas suponen una combinación diferente de los tipos de control generando una estrategia que se apoya, preponderantemente, en el control estructural. Este desarrolla mecanismos que pueden ser entre otros la formalización en los procedimientos administrativos, los requisitos técnicos para la realización de las tareas y el apoyo a la toma de decisión.

Figura 2: Estrategia de Control en Organizaciones Burocráticas

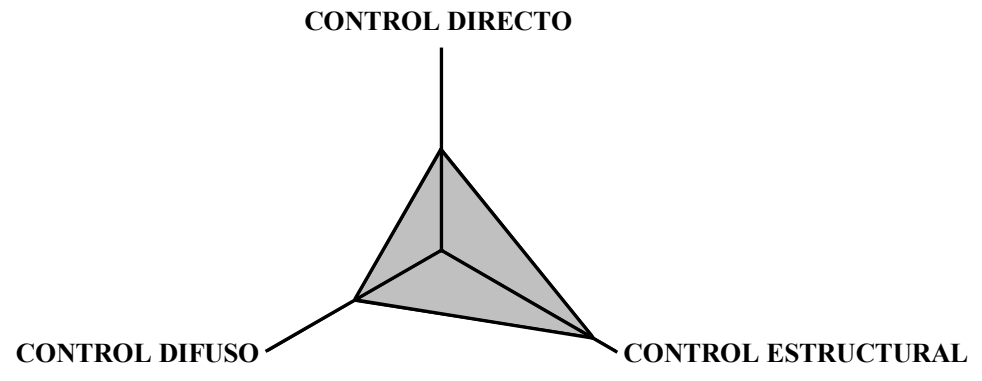

Fuente: elaboración propia.

Las organizaciones de voluntariado y organizaciones no gubernamentales configuran una estrategia de control en la que el control difuso, compuesto por mecanismos como la participación de los miembros en encuentros periódicos, la selección previa de sus participantes voluntarios y la conformación del trabajo a programas de acción previamente definidos, representa la principal forma de control organizacional. 
Figura 3: Estrategia de Control en Organizaciones Normativas

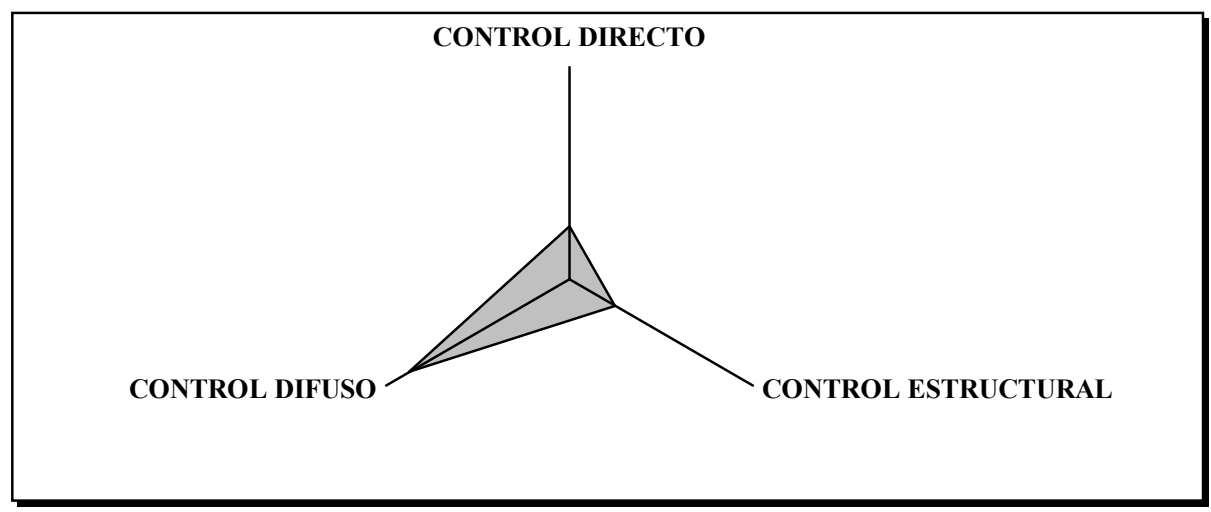

Fuente: elaboración propia.

\section{Diseño y Proceso de InVestigación}

Esta investigación quiere contribuir para una mejor comprensión del fenómeno del control en las ONGs españolas. Para lograr ese objetivo, optó por el modus operandi de un estudio de multi-caso. En concreto los casos estudiados son las ONGs Manos Unidas y la Asociación Andaluza por la Solidaridad y la Paz (ASPA), dedicadas a la cooperación al desarrollo, Amnistía Internacional en la defensa de los derechos humanos y, la Asociación Ecologista de Defensa de la Naturaleza (Aedenat), que actúa en la preservación del medio ambiente.

La elección de un estudio multi-caso exige la aplicación de un diseño metodológico de parámetros semejantes al de un estudio de caso simple. No obstante, según Yin (1994), las conclusiones obtenidas de un estudio de multi-caso pueden ser consideradas más reforzadas y el estudio, en su conjunto, más coherente. Hemos buscado, en esta opción, mayor capacidad de generalización empírica, de verificación y de generación teórica y, por esa razón, las organizaciones fueran seleccionadas en virtud de su capacidad para duplicar las observaciones inferidas a partir del marco de referencia teórico y, de ese modo, reforzar las conclusiones a través de la multiplicación de las evidencias. De esa forma no se ha pretendido contraponer analíticamente los cuatro casos elegidos para estudio, sino que averiguar en cada uno de ellos los mecanismos de ejercicio del control.

El ámbito de estudio de esta investigación circunscríbese a las oficinas centrales en Madrid, Barcelona y Sevilla, España, y algunas de las delegaciones regionales de las cuatro ONGs citadas. Ellas detienen gran representatividad en sus respectivos 
campos de actuación, cuya diversidad permitió no limitar las conclusiones alcanzadas a un campo de acción muy restringido a la vez que, su nivel de formalización hizo posible su clasificación como organizaciones formales y, su peculiar evolución histórica facilitó poner de relieve las influencias ejercidas por el contexto actual.

La población de esta investigación fue compuesta fundamentalmente por los directivos de las ONGs seleccionadas, una muestra de voluntarios estables que desarrollan sus actividades en las oficinas centrales, secciones o delegaciones de las referidas ONGs. En un primer momento fueron realizadas entrevistas semiestructuradas a los directivos con el objetivo de reconocer los elementos considerados importantes en el cotidiano de trabajo y de participación en el interior de las ONGs. En la segunda etapa fueron elaboradas encuestas que, tras el test previo que probó la claridad, precisión y adecuación de las preguntas, fueron aplicadas a una muestra de voluntarios estables de cada unidad de las ONGs en estudio. Además, fueron usados datos secundarios, de fuentes previamente elaboradas por otros, tales como los documentos internos y los textos para divulgación externa publicados por las ONGs.

\section{Caracterización del Sector No Gubernamental Español}

El carácter reivindicativo de su acción marca la identidad de la mayoría de las ONGs que evidencian, en sus comportamientos organizativos, valores contrahegemónicos característicos de los nuevos movimientos sociales (Badelt, 1990). Estos conforman lo que denominamos el entorno alternativo. Es aquí donde se conserva, aún, la capacidad de resistencia del sector no gubernamental a su progresiva institucionalización, fenómeno que marca la actual etapa de la vida de las ONGs españolas (Carvalho, 1997).

La independencia del gobierno, dictada como una característica básica de las ONGs, está amenazada por la creciente dependencia de las subvenciones públicas. La libertad y autonomía casi total del sector parece ahora convivir con una regulación y un ordenamiento jurídico-legal que gradualmente va delimitando los marcos en que se pueden mover las ONGs.

La defensa de su identidad independiente y contestataria, rasgos fundamentales de las organizaciones de los nuevos movimientos sociales (Dalton, Kuechler y Bürklin, 1992), alternan con la búsqueda de la construcción de una imagen de eficiencia (Handy, 1990) ante el público y los elementos claves de los poderes instituidos. 
El contexto institucional que parece emerger de este panel mantiene la fuerza de valores considerados universales como la solidaridad, el voluntariado y la cooperación. Sin embargo se constata el desplazamiento de algunos parámetros en dirección a modelos de estructuración y de gestión del trabajo caracterizados por la complejidad y la formalización de procesos organizativos, la dependencia de instituciones de su entorno y la sumisión a criterios de eficiencia técnica. Estos cambios profundos que se pueden identificar actualmente, parecen ser responsables por alteraciones significativas en la configuración de las ONGs españolas.

\section{Configuración de los Tipos de Control en las ONGs en Estudio}

El control directo comprende mecanismos como la claridad de las órdenes (o de las directrices formuladas), la rigidez de los horarios de trabajo (o participación en el caso de los voluntarios) y la supervisión más directa de las acciones realizadas. Cuanto mayor sean los niveles asignados a estas formas de control ${ }^{(2)}$ (según las informaciones ofrecidas por los voluntarios), mayor será el grado percibido en la adopción de la forma de control directo.

Las características seleccionadas para configurar la forma de control estructural son: la formalización (el conjunto de normas y reglas escritas que conducen y reglamentan las acciones), los requisitos técnicos (representantes del paradigma de la eficiencia, propio del modelo burocrático de gestión), y la jerarquía (igualmente característica vital de la burocracia que añade el concepto de autoridad al rasgo anterior).

El control difuso se inspira en la presión ejercida por las premisas culturales y el comportamiento de los voluntarios. En esta investigación lo componen mecanismos como los programas de acción que, colectivamente elaborados, guían la acción y circunscriben la actuación de los individuos en la organización; la selección previa de los miembros voluntarios de las ONGs cuja intención es garantizar el ajuste entre el background cultural del individuo y la cultura establecida de la organización; y a participación en encuentros, que reúnen periódicamente a los voluntarios de la organización (y cuyo papel preponderante no es la coordinación y el perfeccionamiento técnico, sino el refuerzo cultural y simbólico a través de la confraternización), fue considerada una característica relevante del control difuso en su aplicación particular a las organizaciones voluntarias.

Según los datos recogidos en las organizaciones seleccionadas ${ }^{(3)}$, sus directivos y voluntarios con participación de carácter permanente, plasmada en la Figura 
4 y detallada en el Cuadro 2, revela el predominio del control difuso sobre los demás.

\section{Figura 4: Configuración de Control en las Cuatro ONGs}

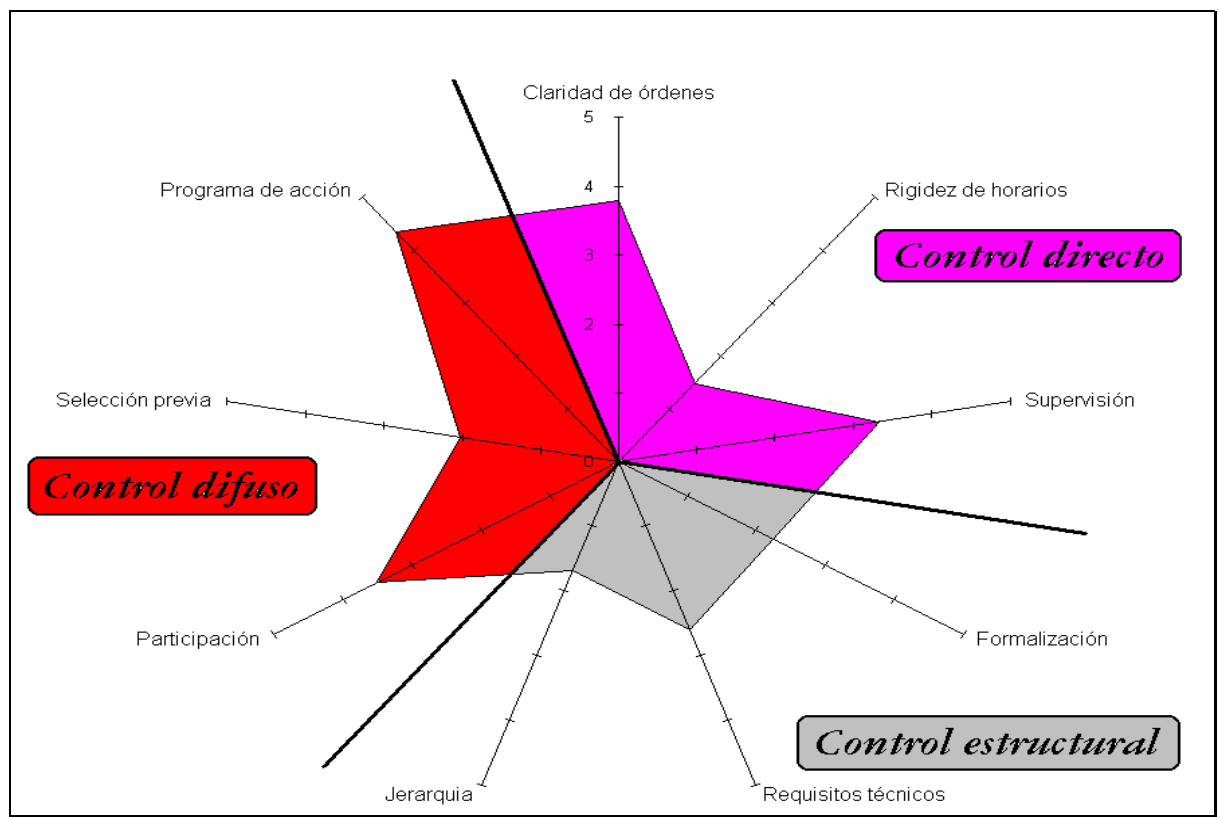

Fuente: encuestas realizadas.

La forma de control difuso predomina sobre el resto de las formas de control contempladas. En el modelo difuso, el principal mecanismo destacado es la determinación de la acción administrativa mediante la definición de programas de acción. Su objetivo declarado es trazar la acción organizativa que se va a realizar, controlando la actuación de los voluntarios mediante la limitación de determinados parámetros.

La participación, en encuentros y otros tipos de acciones colectivas, constituye el segundo mecanismo de control más usual. Con esta valoración se resalta el aspecto simbólico al privilegiarse los valores, símbolos y rituales compartidos, una práctica que la observación de la vida de la ONG identifica como central.

La selección previa de los voluntarios registra una valoración menor. Sin embargo, en el análisis individual de cada ONG se han podido verificar diferencias significativas entre los niveles indicados para este mecanismo. Mientras que en ONGs grandes que demostran un fuerte proceso de institucionalización, como Manos Unidas y Amnistía Internacional, se considera como una práctica 
significativa, en ASPA y Aedenat, con características más alternativas, la selección previa de los candidatos a voluntarios se percibe como un mecanismo casi inexistente.

\section{Cuadro 2: Medias de Ocurrencia de Control}

\begin{tabular}{|l|c|c|c|c||}
\hline Mecanismos de control & Aedenat & Manos Unidas & Amnistia Intern. & ASPA \\
\hline Claridad de órdenes & 3,5 & 3,9 & 3,9 & 3,8 \\
\hline Rigidez de horarios & 1,5 & 1,4 & 1,4 & 1,5 \\
\hline Supervisión & 3,2 & 3,3 & 3,4 & 3,3 \\
\hline Control Directo & 2,74 & 2,91 & 2,90 & 2,89 \\
\hline Formalización & 2,4 & 1,4 & 3,2 & 2,0 \\
\hline Requisitos técnicos & 2,8 & 1,8 & 3,0 & 2,7 \\
\hline Toma de decisión & 1,3 & 2,4 & 1,7 & 1,3 \\
\hline Control Estructural & 2,18 & 1,85 & 2,65 & 2,0 \\
\hline Participación encuentros & 3,7 & 3,0 & 3,5 & 3,9 \\
\hline Selección & 1,6 & 2,5 & 2,4 & 1,5 \\
\hline Programas de acción & 4,3 & 4,2 & 4,5 & 4,4 \\
\hline Control Difuso & 3,19 & 3,2 & 3,47 & 3,29 \\
\hline
\end{tabular}

Fuente: encuestas realizadas.

De acuerdo con el modelo teórico sugerido y confirmado en esta verificación empírica, las formas de control difuso constituyen el modelo típico, y por lo tanto predominante, en las ONGs. El control difuso está asociado, según nuestra propuesta, al entorno alternativo en cuyo seno se preservan los valores contrahegemónicos y, por lo tanto, esta constatación nos permite confirmar la permanencia del carácter alternativo y contestatario de estas organizaciones.

Sin embargo, la adopción, en niveles bastante elevados, de formas de control como la claridad de las órdenes, la rigidez de horarios y la supervisión del trabajo, configurando estrategias de control de tipo directo, evidencia el progresivo desvío de las ONGs hacia prácticas de gestión propias de las organizaciones con ánimo de lucro. La progresión es percibida por estas formas de control obteneren mayores niveles de frecuencia en la ONG más formalizada y de mayor tamaño (Manos Unidas) y menores niveles de frecuencia en la ONG más joven y menos formalizada.

De acuerdo con el modelo teórico propuesto, las formas de control directo se manifiestan sobre todo en las organizaciones tayloristas que, apoyadas en el paradigma del mercado, se enfrentan a la competencia por los recursos escasos y actúan de modo simple y concreto para sobrevivir. Pero, en el caso de las ONGs, la adopción de estas características de la gestión privada y la presión del entorno a través de la reglamentación y la dependencia financiera y jurídica de los poderes instituidos parece incidir en la configuración de las estrategias de control. La 
presión por eficiencia y eficacia en la acción parece exigir la incorporación de técnicas, que la gestión de las organizaciones con ánimo de lucro ya ha demostrado como válidas.

Los mecanismos estructurales de control, a su vez, exigen, para su adopción, un mayor nivel de estructuración burocrática en comparación con los mecanismos de control directo. El control estructural constituye el soporte formal para la toma de decisiones lo que implica la utilización de mecanismos indirectos. En todas las ONGs, esta forma de control registró valores con poca significación, resultado que se mantiene en la perspectiva global.

Un análisis detallado muestra que los requisitos técnicos registran un nivel medio de significación: el carácter amateur de la acción está dejando paso al rigor y a la calidad. La formalización registró un nivel medio/bajo aunque esté por encima de lo esperado, pero inferior a lo que debería ocurrir en una organización burocrática. El concepto de jerarquía está cargado negativamente de lo que habitualmente se entiende por burocracia. Por tanto, el rechazo registrado en este mecanismo de control estructural resulta comprensible.

Las formas de control estructural representan el modo hegemónico que caracteriza las organizaciones burocráticas. Estos mecanismos suponen un nivel superior de estructuración burocrática en comparación con los mecanismos simples del control directo. Así, resultó comprensible esperar que fuera el tipo de control menos característico en las organizaciones no gubernamentales cuya dimensión informal tiene un peso relativamente importante.

\section{Conclusiones}

Como hemos resaltado, no está todavía construido un cuerpo sólido y coherente de conceptos, en el campo del análisis económico y organizacional, que sea capaz de interpretar las ONGs en una dimensión cuyo centro epistemológico no esté sujeto en la interpretación del Estado o del mercado.

Esta investigación quiso contribuir en esa tarea y, así, tuvo como objetivo presentar un modelo de análisis del control, adecuado a una aplicación empírica a organizaciones particulares: las organizaciones no gubernamentales, en el contexto actual de la realidad del sector no gubernamental español.

Hemos percibido que, confirmando la propuesta del modelo teórico, la aplicación empírica verificó una predominancia de los mecanismos perfectamente discretos de control, configurando una estrategia basada en el control difuso en las ONGs. 
Valores, símbolos y rituales fueron revelados elementos importantes en ese mapa organizativo.

Sin embargo, en su conjunto, las formas de control de las cuatro ONGs han señalado una estrategia donde se percibe un progresivo aumento de los mecanismos de control directo, aunque predominen las formas difusas.

Esta verificación permitió observar una diferencia entre el modelo teórico expuesto y el estudio empírico: los datos arrojan unos niveles de control directo superiores a los esperados en todas las organizaciones investigadas, aunque, las más antiguas, estructuradas y competitivas revelen mayores niveles de frecuencia de control directo. Atribuimos la razón de esta divergencia a la influencia ejercida por el contexto institucional sobre las organizaciones no gubernamentales. Esa influencia se registra en los nuevos procedimientos de financiación pública, el ordenamiento jurídico en progresiva construcción y la competencia creada en un sector que creció de forma explosiva.

Partiendo de esta base, podemos inferir que los cambios en las exigencias reguladoras de las administraciones públicas, en el ordenamiento jurídico y en las formas de financiación de las organizaciones no gubernamentales habrán tenido efectos en las estructuras burocráticas de esas organizaciones. Estos cambios, producidos en el entorno institucional, afectaron las características estructurales y repercutieron en la configuración de las estrategias de control de las organizaciones.

Estructurada de este modo, la investigación habrá permitido un acercamiento al fenómeno expuesto, que podrá constituir una ayuda significativa para una mejor comprensión a la problemática de las organizaciones no gubernamentales en España. Al valor intrínseco que representa conocer un fenómeno social, se añade la relevancia creciente que estas organizaciones han demostrado tener en la sociedad.

Este es el panorama de las organizaciones no gubernamentales que vislumbra un sector dividido entre, por un lado, las presiones del entorno institucional en pos de la modernización, la eficiencia y la excelencia empresarial, y por otro, la preservación de su identidad como sector independiente y autónomo de los poderes instituidos.

\section{Notas}

${ }^{1}$ Este trabajo deriva de la tesis de doctorado titulada EI control organizativo en organizaciones no gubernamentales: una perspectiva institucional, leída por la autora en noviembre de 1997, en la Universidad de Córdoba, España. 
${ }^{2}$ Los niveles de frecuencia percibidos de cada mecanismo de control, averiguado en las encuestas, fueron medidos con base en una escala Likert cuyo abanico iba desde muy bajo hasta muy alto.

${ }^{3}$ Aquí presentaremos tan solamente los resultados de la investigación realizada en las cuatro ONGs, tomadas en su conjunto, aunque el análisis original haya sido realizado en cada organización separadamente.

\section{ReFERENCIAS BibLIOGRÁfiCAS}

AHRNE, G.

Agency and organization : toward an organizational theory of society. London: Sage Publications, 1990.

BADELT, C.

Institutional choice and the nonprofit sector. In: ANHEIER, H. K.; SEIBEL; W. (Eds.). The third sector: comparative studies of nonprofit organizations. New York : Walter de Gruyter, 1990.

BURRIS, B. H.

Technocratic organization and control. Organizations Studies, v. 10, n. 1, p. 01-22, 1989.

CARVALHO, C. A. P. DE. El control organizativo en
organizaciones
gubernamentales : perspectiva institucional. Córdoba, 1997. Tesis (Doctorado) Universidad de Córdoba, España.

CLEGG, S.;

DUNKERLEY, D.

Organizations, class and control.2.ed. London : Routledge, 1991.

COURPASSON, D.

Régulation et gouvernement des organisations - pour une sociologie de l'Action managériale. Sociologie du Travail, v. XXXIX, n. 1, p. 39-61, 1997.

DALTON, R. J.;

KUECHLER, M.;

BÜRKLIN, W.

El reto de los nuevos movimientos. In: DALTON, R. J.; KUECHLER, M. (Eds.). Los nuevos movimientos sociales. València : Edicions Alfons el Magnànim/ Generalitat Valenciana, 1992.

DIMAGGIO, P. J.;

ANHEIER. H. K.

The sociology of nonprofit organizations and sectors. Annual Review of Sociology, v. 16, p. 137-59, 1990.

EDWARDS, R.

Contested terrain. 2.ed. London: Heinemann, 1980. 
HANDY, C.

Understanding voluntary organizations. London: Penguin Books, 1990.

HARDY, C.;

CLEGG, S. R.

Some dare call it power. In: CLEGG, S. R.; HARDY, C.; NORD, W. R. (Eds.). Handbook of organization studies. London: Sage Publications, 1996.

HERMAN, A.

Conceptualizing control : domination and hegemony in the capitalist labor process. The Insurgent Sociologyst, v. 11, n. 3, p. 07-22, 1982.

MEYER, J. W.;

ROWAN, B.

Institutionalized organizations : formal structure as myth and ceremony. In: MEYER, J. W.; SCOTT, W. R. (Eds). Organizational environments : ritual and rationality. upd. ed. London : Sage Publications, 1992.

\section{MORGAN, G.}

Images of organizations. 2. ed. London : Sage Publications, 1997.
PÉREZ VILARIÑO, J.;

SCHOENHERR, R. A.

Racionalidad y control en las organizaciones complejas. Revista Española de Investigaciones Sociologicas, v. 39, p. 119-39, 1987.

PERROW, C.

Sociología de las organizaciones. 3.ed.New York : McGraw-Hill, 1990.

RUIZ OLABUENAGA, J. I.

Sociología de las organizaciones. Bilbao : Universidad de Deusto, 1995.

SCOTT, W. R.

The adolescence of institutional theory. Administrative Science Quaterly, v. 32, n. 4, p. 493-511, 1987.

Institutions and organizations. London : Sage Publications, 1995.

YIN, R. K.

Case study research: design and methods. 2. ed. London : Sage Publications, 1994.

WEBER, M.

Economía y sociedad. Madrid : Fondo de Cultura Económica, 1993. 